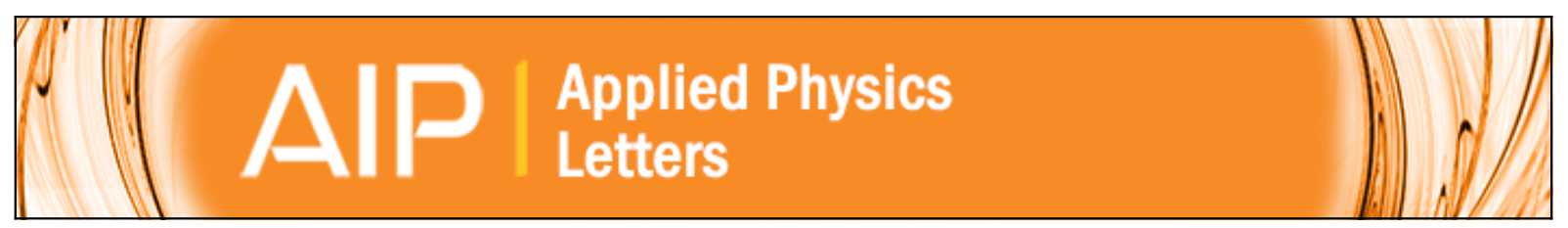

\title{
Ultrafast carrier dynamics in gold/iron-oxide nanocrystal heterodimers
}

Kseniya Korobchevskaya, Chandramohan George, Alberto Diaspro, Liberato Manna, Roberto Cingolani, and Alberto Comin

Citation: Applied Physics Letters 99, 011907 (2011); doi: 10.1063/1.3609324

View online: http://dx.doi.org/10.1063/1.3609324

View Table of Contents: http://scitation.aip.org/content/aip/journal/apl/99/1?ver=pdfcov

Published by the AIP Publishing

\section{Articles you may be interested in}

Scanning microwave microscopy and scanning capacitance microscopy on colloidal nanocrystals

J. Appl. Phys. 109, 064313 (2011); 10.1063/1.3553867

Ultrafast dynamics of femtosecond laser-induced nanostructure formation on metals

Appl. Phys. Lett. 95, 123111 (2009); 10.1063/1.3222937

Ultrafast dynamics of photoexcited charge carriers in nanocrystalline diamond Appl. Phys. Lett. 93, 083102 (2008); 10.1063/1.2970962

Synthesis and photoexcited charge carrier dynamics of $\beta$ - Fe O O H nanorods Appl. Phys. Lett. 90, 103504 (2007); 10.1063/1.2711395

Ultrafast carrier dynamics in $\mathrm{ZnO}$ nanorods

Appl. Phys. Lett. 87, 023106 (2005); 10.1063/1.1989444

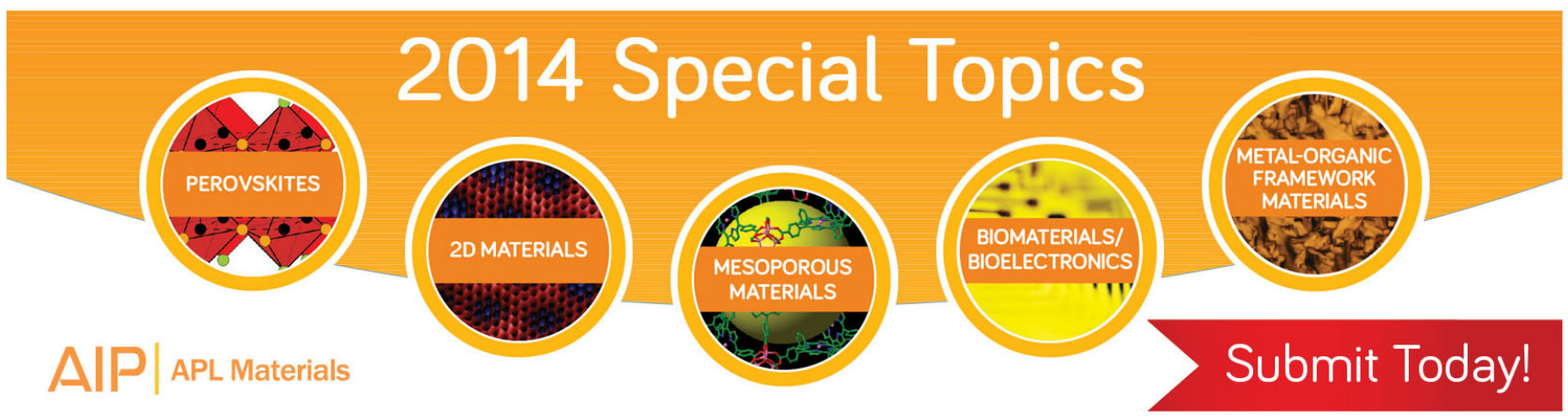




\title{
Ultrafast carrier dynamics in gold/iron-oxide nanocrystal heterodimers
}

\author{
Kseniya Korobchevskaya, Chandramohan George, Alberto Diaspro, Liberato Manna, \\ Roberto Cingolani, and Alberto Comin ${ }^{\text {a) }}$ \\ NanoPhysics and NanoChemistry Division, Istituto Italiano di Tecnologia, 16136 Genova, Italy
}

(Received 20 April 2011; accepted 18 June 2011; published online 7 July 2011)

Colloidal nanocrystal heterodimers composed of a gold domain and an iron oxide domain have been investigated by femtosecond transient absorption spectroscopy. The measured decay times were compared with the ones obtained from samples of "only" gold nanocrystals and iron oxide nanocrystals. Our results indicate that there is no significant charge transfer at the interface between gold and iron oxide in heterodimers. (C) 2011 American Institute of Physics. [doi:10.1063/1.3609324]

Iron oxide nanocrystals $\left(\mathrm{Fe}_{3} \mathrm{O}_{4}\right.$ and $\left.\gamma-\mathrm{Fe}_{2} \mathrm{O}_{3}\right)$ have widely been used in various biomedical applications, for example as contrast agents in magnetic resonance imaging (MRI) and in hyperthermia therapy. ${ }^{1,2}$ However, for photoelectrochemical applications, the use of iron oxide nanocrystals has been limited by their low conductivity and low absorption quantum efficiency. On the other hand, $\alpha$-iron oxide nanoparticles decorated with gold domains have been exploited as electrodes and exhibited enhanced catalytic properties, as observed by Thimsen et al. ${ }^{3}$ who also suggested that either energy transfer or charge-transfer between gold and iron oxide might be responsible for such enhancement. More in general, colloidal Au-iron oxide nanocrystal heterostructures have received considerable attention recently, because they exhibit both magnetic and plasmonic properties and have been proposed for many applications in biology ${ }^{4}$ and in catalysis. ${ }^{3}$ To date, many reports have been published on the synthesis of Au-iron oxide hetero-nanostructures, and some of them are cited here: $\sim 12 \mathrm{~nm} \mathrm{Fe}_{3} \mathrm{O}_{4}$ $\mathrm{Au}$ core-shell nanocrystals by $\mathrm{Xu}$ et al. ${ }^{5}{ }^{\mathrm{Au}} \mathrm{A}-\mathrm{Fe}_{3} \mathrm{O}_{4}$ dumbbells of a variety of sizes $(\sim 10-24 \mathrm{~nm})$ by $\mathrm{Yu}$ et al.; ${ }^{6}$ core/ shell nanocrystals with varying thickness of the iron oxide shell (from $\sim 2.5$ to $3.3 \mathrm{~nm}$ ) by Shevchenko et al. $;^{4}$ and dumb-bell nanocrystals $(\sim 20 \mathrm{~nm})$ with two iron oxide domains by Shi et $a l^{7}$

Various works have reported also a significant red-shift in the gold plasmon peak in $\mathrm{Au}-\gamma-\mathrm{Fe}_{2} \mathrm{O}_{3}$ nanocrystal heterostructures with respect to its position in pure Au nanoparticles. This red shift has been generally ascribed to a change in dielectric constant of the surrounding medium due to the presence of iron oxide, although Yu et al. ${ }^{6}$ and Wang et al. ${ }^{8}$ proposed that this shift could be also due to electron deficiency in gold, as a result of a possible transfer of electrons to iron oxide. Interestingly, Shi et al. ${ }^{7}$ too observed a plasmonic peak at $570 \mathrm{~nm}$ (i.e., considerably red-shifted from $520 \mathrm{~nm}$ ) in comparable sizes of nanoparticles, but they ruled out the charge-transfer hypothesis as they estimated that it would require a $20 \%$ reduction of the gold free electron density to justify such shift.

We report here time-resolved spectroscopic measurements on Au nanocrystals ( $10 \mathrm{~nm}$ diameter), $\mathrm{Fe}_{2} \mathrm{O}_{3}$ nanocrystals (20 nm diameter), and on $\mathrm{Au}-\mathrm{Fe}_{2} \mathrm{O}_{3}$ nanocrystal

\footnotetext{
a)Electronic mail: alberto.comin@iit.it.
}

heterodimers, with the aim to elucidate electron transfer processes at the $\mathrm{Au}-\mathrm{Fe}_{2} \mathrm{O}_{3}$ interface in $\mathrm{Au}-\mathrm{Fe}_{2} \mathrm{O}_{3}$ nanocrystals and their influence on the plasmonic behavior of the Au domain. In a typical heterodimer, the iron oxide shell was grown on a gold nanocrystal seed and evolved upon annealing into an asymmetric dumb-bell shape, as shown in Fig. 1. By comparing the dynamics of $\mathrm{Au}-\mathrm{Fe}_{2} \mathrm{O}_{3}$ heterodimers with that of $\mathrm{Au}$ only and $\mathrm{Fe}_{2} \mathrm{O}_{3}$ only nanoparticles, we could safely exclude any significant charge transfer from the gold domain to the iron oxide domain in the heterodimers. Despite $\mathrm{Au}$ and $\mathrm{Fe}_{2} \mathrm{O}_{3}$ nanocrystals had been investigated quite extensively, to the best of our knowledge, this is the first transient-absorption study on $\mathrm{Au}-\mathrm{Fe}_{2} \mathrm{O}_{3}$ nanocrystal heterodimers.

The various nanocrystals reported in this study were synthesized following George et al. ${ }^{9}$ In particular, the gold/ iron oxide asymmetric heterodimers $(20 \mathrm{~nm})$ were prepared by decomposing iron pentacarbonyl on gold nanocrystals via high-temperature colloidal synthesis. The morphological characterization of the particles was carried out via transmission electron microscopy (TEM) using a JEOL JEM 1001 microscope. In Fig. 1, we show a TEM image of the typical gold/iron oxide heterodimers. The absorption spectra of the nanocrystals were recorded in toluene using a Varian Cary 5000UV-vis-Near Infrared (NIR) spectrophotometer, with 1 $\mathrm{cm}$ quartz cells. Transient absorption experiments were performed with a pump-probe setup based on a Ti:Sapphire femtosecond laser $(800 \mathrm{~nm}$, repetition rate $1 \mathrm{KHz}$, and pulse width $50 \mathrm{fs}$ ). The main beam was split into three parts: (1) probe: $1 \mathrm{~mW}, 800 \mathrm{~nm}$, (2) reference: identical to probe, and (3) pump: $10 \mathrm{~mW}, 400 \mathrm{~nm}$, obtained by frequency doubling in a $0.2 \mathrm{~mm}$ thick Beta Barium Borate (BBO) crystal. Probe spot size was $0.5 \mathrm{~mm}$ and energy fluence was $30 \mu \mathrm{J} / \mathrm{cm}^{2}$.

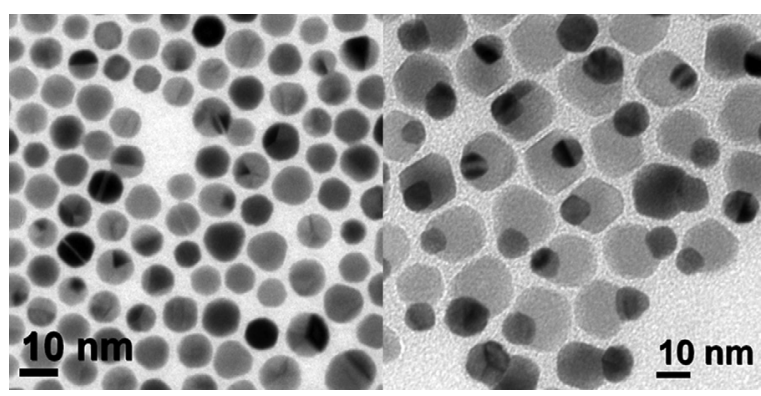

FIG. 1. Transmission electron microscopy images of Au (left) and gold/iron oxide nanocrystals (right). 
The pump was chopped at $500 \mathrm{~Hz}$ and focused to $1 \mathrm{~mm}$ spot in order to obtain $0.3 \mathrm{~mJ} / \mathrm{cm}^{2}$ energy density on the sample. The difference between the intensities of the transmitted probe and reference beams was detected by a matched photodiode pair. The sample was loaded in a $1 \mathrm{~mm}$ quartz cuvette. By comparing TEM images of the samples before and after experiments, we verified that there was no sign of laser induced melting or nanoparticle aggregation.

Steady state absorption spectra of the three samples are reported in Fig. 2. Iron oxide exhibits a featureless spectrum, characterized by increasing absorption for photon energies above the $\gamma-\mathrm{Fe}_{2} \mathrm{O}_{3}$ band-gap $(2.0 \mathrm{eV}$ equivalent to 611 $\mathrm{nm}){ }^{10-12}$ The spectrum of gold nanocrystals shows a plasmonic resonance at $525 \mathrm{~nm}$. The position of the peak is known to depend on several factors, such as the density of the conduction electrons and the nanoparticles surrounding medium, as described by the Mie theory. ${ }^{13}$ For wavelengths shorter than $540 \mathrm{~nm}$, inter-band $5 \mathrm{~d} \rightarrow 6$ s transitions become more significant. ${ }^{14}$ The gold plasmon peak in the sample of gold/iron oxide heterodimers is significantly red-shifted with respect to that of the sample of gold only nanoparticles (Fig. 2), which is in agreement with several reports. ${ }^{5,15,16} \mathrm{~A}$ change of the dielectric function of the environment around the gold nanocrystals can clearly induce such a shift, in accordance with Mie theory. ${ }^{13}$ Under the dipolar approximation, the absorption cross section of a spherical particle, whose size is much smaller than the wave-length of the incident light, has a resonance given by $\operatorname{Re}[\varepsilon(\lambda)]=-2 \varepsilon_{\text {media }}$ $=-2 n_{\text {media }}^{2}$. This means that already by changing solvent from water $\left(\mathrm{n}_{\text {media }}=1.33\right)$ to toluene $\left(\mathrm{n}_{\text {media }}=1.50\right),{ }^{17}$ we can expect a shift of the plasmon peak from $522 \mathrm{~nm}$ to $536 \mathrm{~nm}$. Since the dielectric permittivity of iron oxide $\left(\mathrm{n}_{\text {media }}=2.6\right)($ Ref. 18) is higher than that of toluene, even larger shifts are expected. In the present case, we modeled for simplicity the heterodimers as core-shell particles with effective coating thickness $t_{\text {eff. }}$. We used wavelength dependent complex refractive indexes for both gold and iron oxide ${ }^{19}$ and a wavelength dependent real refractive index for toluene. We found that an effective thickness of $3 \mathrm{~nm}$ for the iron oxide shell on gold could account for the observed red-shift. However, to match precisely the measured plasmonic frequency, computation approaches based of finite elements or T-matrix method ${ }^{20}$ would be required.

An alternative explanation for the shift in the gold plasmon peak has been proposed by Daniel and Astruc ${ }^{21}$ Since

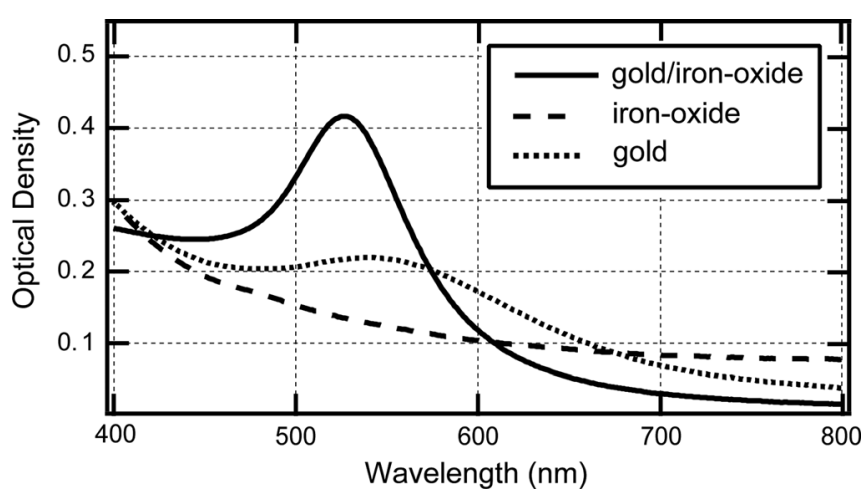

FIG. 2. UV-vis spectra of the $\mathrm{Au}$ (solid line), $\mathrm{Au}-\mathrm{Fe}_{2} \mathrm{O}_{3}$ dimers (dotted line), and $\mathrm{Fe}_{2} \mathrm{O}_{3}$ nanoparticle (dashed line) dispersed in toluene. in the dipole approximation the plasmon frequency is proportional to the square root of the free-electron density, the transfer of an electron from the gold domain to iron oxide domain could also explain the observed red shift in the heterodimers. By using Mie theory, we estimated that about $6 \%$ of the free electrons should transfer from gold to iron oxide in order to cause the observed red-shift, that is, from $525 \mathrm{~nm}$ to $542 \mathrm{~nm}$. It can be noted that the fraction of the atoms which resides on the particle surface can be estimated by the following relationship: ${ }^{22} P=4 / N^{-1 / 3} \cdot 100$, where $\mathrm{N}$ is the total number of atoms of the particle. According to this formula, for a $10 \mathrm{~nm}$ diameter gold nanocrystal, about $13 \%$ of the atoms resides at the interface, ${ }^{22}$ where the gold electrons could be trapped by many $\mathrm{Fe}^{3+}$ defect states. ${ }^{23}$ Therefore, the sole analysis of static absorption spectra cannot explain the origin of the plasmon red-shift in dimers.

In Figs. 3 and 4, we report the transient absorption curves for gold, iron oxide, and gold-iron oxide dimers. Both figures contain the same data, plotted in different time ranges in order to illustrate the short and long dynamics. To quantify the relevant time scales, the data were analyzed in two steps. First, we fitted the $0-20$ ps interval using a linear combination of two exponentials, then we fitted the full time range using a triple exponential with the first two time constants fixed as parameters.

The experimental conditions were identical for all samples. The nanocrystals were dissolved in toluene (relatively low optical density: 0.02 at $800 \mathrm{~nm}$ ). At short time delays, the Au pump-probe signal (Fig. 3(a)) could be modeled by a single exponential decay with time constant $1.1 \pm 0.1 \mathrm{ps}$, which matches the known value for the electron-phonon coupling

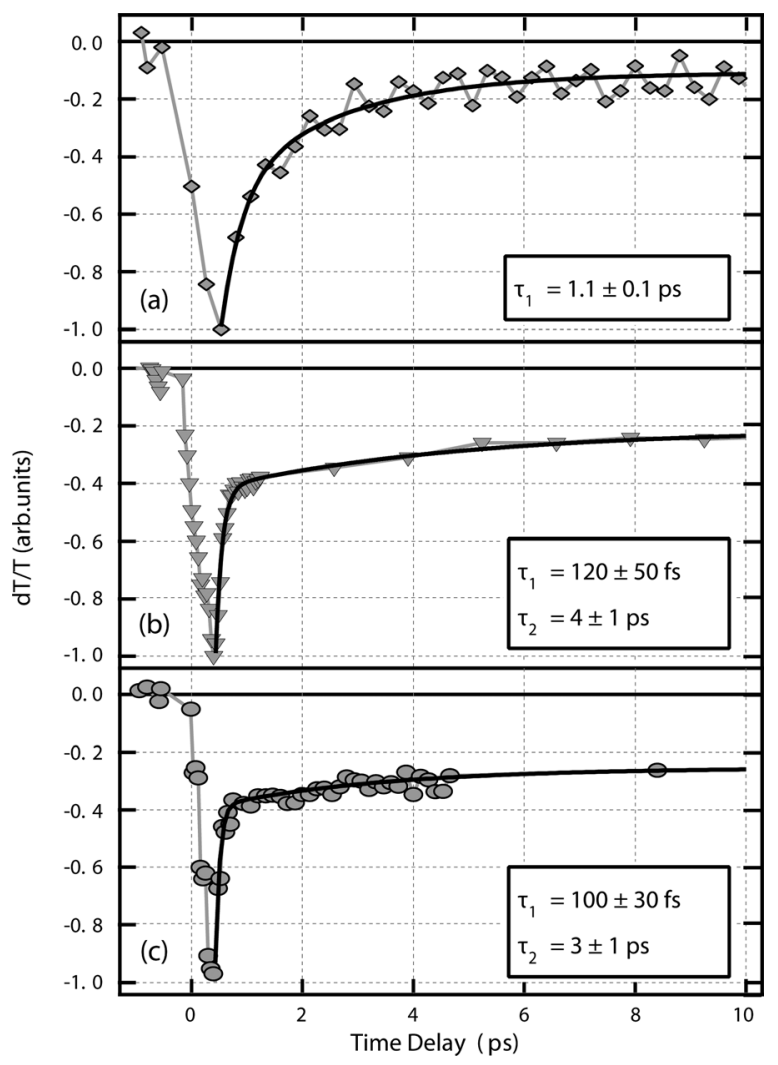

FIG. 3. Transient absorption traces of $10 \mathrm{~nm}$ gold (a), $20 \mathrm{~nm}$ iron oxide (b), and gold/iron oxide heterodimers (c). 


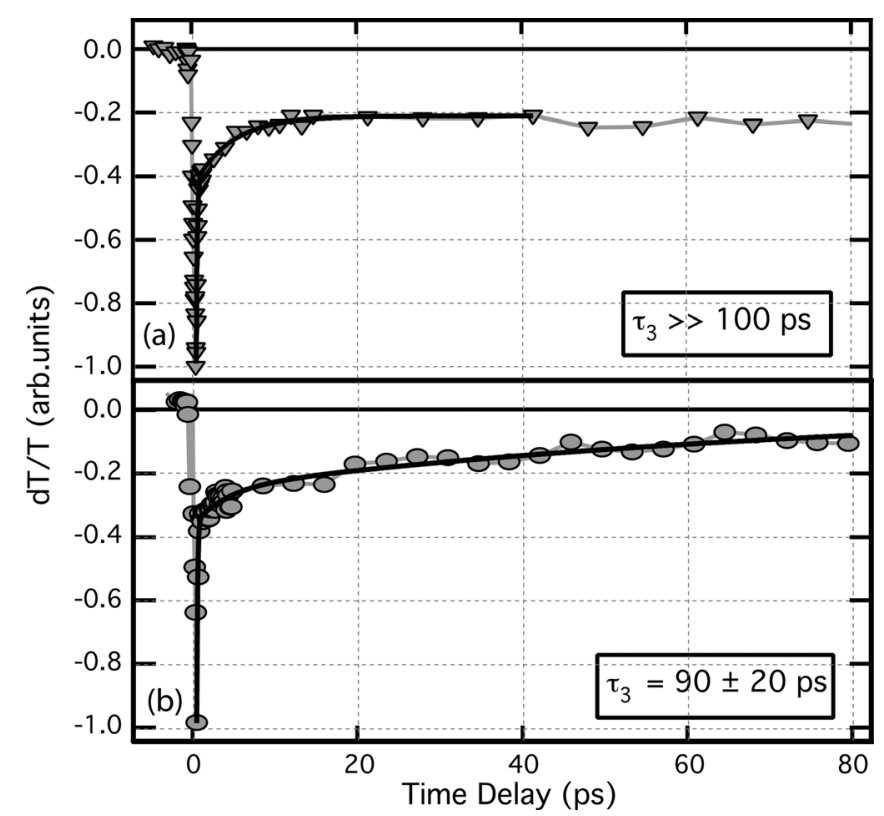

FIG. 4. Long time component of the transient absorption traces of iron oxide (a) and gold/iron-oxide nanocrystals (b).

time. ${ }^{24,25}$ The error-bar was determined as standard deviation of fitted decay constants from multiple experiments.

The transient absorption signal for the iron oxide only nanoparticles is reported in Figs. 3(b) (short time range) and 4(a) (long time range). The excitation beam at $400 \mathrm{~nm}$ creates a hot-electron population which rapidly relaxes to the bottom of the conduction band (about $100 \mathrm{fs}$ ) via electronelectron scattering. However, according to Cherepy et al., ${ }^{23}$ the pump-probe signal at this time scale does not reflect the conduction electrons dynamics but rather a fast electron trapping. Recombination of the excited carriers can happen either radiatively or not-radiatively via mid-gap defect states. In $\gamma$-iron oxide nanocrystals, the non-radiative channel is dominant, because of the numerous trap states present, as confirmed by the very low florescence quantum yield of these particles. ${ }^{23}$ Our data could be well fitted by a combination of three exponentials, with $\tau_{1}=120 \pm 50 \mathrm{fs}, \tau_{2}=4 \pm 1$ ps, and $\tau_{3}>100 \mathrm{ps}$, which agrees with what observed by Cherepy et al. in $\mathrm{Fe}_{2} \mathrm{O}_{3}$ nanocrystals (NCs). ${ }^{23}$

In Figs. 3 and 4, we report transient absorption measurements on gold/iron oxide dimers. In principle, the dynamics should represent a super-position of $\mathrm{Au}$ and $\mathrm{Fe}_{2} \mathrm{O}_{3}$ signals, modified by the mutual interactions. By fitting the data with a triple exponential decay function, we found $\tau_{1}=100 \pm 30$ fs, $\tau_{2}=3 \pm 1 \mathrm{ps}$, and $\tau_{3}=90 \pm 20$ ps. It can be seen that short-time decay constants of dimers and iron oxide single particles are very similar. This means that the dynamics is dominated by the iron oxide domain, with a negligible interaction between the iron oxide and $\mathrm{Au}$ domains, and that there is a negligible charge transfer at the interface. Since the iron oxide dynamics on the picosecond time scale is dominated by defect states, ${ }^{23}$ it can be expected that electrons coming from gold domain would partly saturate these defects, modifying the relaxation time-constants. As this effect was not observed, there was no evidence of significant electron transfer between gold and iron oxide. We are therefore able to confirm that the red-shift in the absorption spectra in Au$\mathrm{Fe}_{2} \mathrm{O}_{3}$ dimers is not caused by electron deficiency in gold, but is a dielectric effect induced by the presence of iron oxide as surrounding media. Further studies are however needed to quantify the amount of $\mathrm{Fe}^{3+}$ defect states in iron oxide nanocrystals. It is conceivable that, should their number be too high, the saturation effect caused by the gold electrons would be too small to be observed in the present experimental conditions.

In conclusion, we investigated colloidal nanocrystal heterodimers composed of gold and iron oxide domains by femtosecond transient absorption measurements to verify the proposed hypothesis of charge transfer between gold and iron oxide. We compared the relaxation dynamics of the heterodimers with that of gold only and iron oxide only nanocrystals. We found that the fitted decay time constants of the dimers were very similar to those of the iron oxide nanoparticles, which allowed us to exclude a significant charge transfer at the $\mathrm{Au}-\mathrm{Fe}_{2} \mathrm{O}_{3}$ interface.

${ }^{1}$ A. Figuerola, R. Di Corato, L. Manna, and T. Pellegrino, Pharmacol. Res. 62, 126 (2010).

${ }^{2}$ M. Kettering, H. Zorn, and S. Bremerstreck, Phys. Med. Biol. 54, 5109 (2009).

${ }^{3}$ E. Thimsen, F. Le Formal, M. Grätzel, and S. C. Warren, Nano Lett. 11, 35 (2011).

${ }^{4}$ E. V. Shevchenko, M. I. Bodnarchuk, M. V. Kovalenko, D. V. Talapin, R. K. Smith, S. Aloni, W. Heiss, and A. P. Alivisatos, Adv. Mater. 20, 4323 (2008).

${ }^{5}$ Z. Xu, Y. Hou, and S. Sun, J. Am. Chem. Soc. 129, 8698 (2007).

${ }^{6}$ H. Yu, M. Chen, P. M. Rice, S. X. Wang, R. L. White, and S. Sun, Nano Lett. 5, 379 (2005).

${ }^{7}$ W. Shi, H. Zeng, Y. Sahoo, T. Y. Ohulchanskyy, Y. Ding, Z. L. Wang, M. Swihart, and P. N. Prasad, Nano Lett. 6, 875 (2006).

${ }^{8}$ C. Wang, C. Xu, H. Zeng, and S. Sun, Adv. Mater. 21, 3045 (2009).

${ }^{9}$ C. George, D. Dorfs, G. Bertoni, A. Falqui, A. Genovese, T. Pellegrino, A. Roig, A. Quarta, R. Comparelli, M. L. Curri, R. Cingolani, and L. Manna, J. Am. Chem. Soc. 133, 2205 (2011).

${ }^{10}$ R. M. Cornell and U. Schwertmann, The Iron Oxides: Structure, Properties, Reactions, Occurrences, and Uses, 2nd ed. (Wiley, Weinheim, 2003).

${ }^{11}$ R. Grau-Crespo, A. Y. Al-Baitai, I. Saadoune, and N. H. De Leeuw, J. Phys.: Condens. Matter 22, 255401 (2010).

${ }^{12}$ M. I. Litter and M. A. Blesa, Can. J. Chem. 70, 2502 (1992).

${ }^{13}$ C. F. Bohren and D. R. Huffman, Absorption and Scattering of Light by Small Particles (Wiley, New York, 1983).

${ }^{14}$ C. Voisin, D. Christofilos, P. Loukakos, N. Del Fatti, F. Vallée, J. Lermé, M. Gaudry, E. Cottancin, M. Pellarin, and M. Broyer, Phys. Rev. B 69, 1 (2004).

${ }^{15}$ Y. Wei, R. Klajn, A. O. Pinchuk, and B. A. Grzybowski, Small 4, 1635 (2008).

${ }^{16}$ H. Zeng and S. Sun, Adv. Funct. Mater. 18, 391 (2008).

${ }^{17}$ A. Samoc, J. Appl. Phys. 94, 6167 (2003).

${ }^{18}$ D. R. Lide, ed., CRC Handbook of Chemistry and Physics, 87 th ed. (CRC, Boca Raton, FL, 2007).

${ }^{19}$ M. J. Weber, Handbook of Optical Materials (CRC, Boca Raton, 2003).

${ }^{20}$ M. Mishchenko, J. Quant. Spectrosc. Radiat. Transf. 55, 535 (1996).

${ }^{21}$ M.-C. Daniel and D. Astruc, Chem. Rev. 104, 293 (2004).

${ }^{22}$ C. Rao, P. Thomas, and G. Kulkarni, Nanocrystals: Synthesis, Properties and Applications (Springer, Berlin, 2007).

${ }^{23}$ N. J. Cherepy, D. B. Liston, J. A. Lovejoy, H. Deng, and J. Z. Zhang, J. Phys. Chem. B 102, 770 (1998).

${ }^{24}$ J. H. Hodak, I. Martini, and G. V. Hartland, J. Phys. Chem. B 102, 6958 (1998).

${ }^{25}$ S. L. Logunov, T. S. Ahmadi, M. A. El-Sayed, J. T. Khoury, and R. L. Whetten, J. Phys. Chem. B 101, 3713 (1997). 